IJMMS 32:3 (2002) 129-138

PII. S0161171202012425

http://ijmms.hindawi.com

(c) Hindawi Publishing Corp.

\title{
ON INCLUSION RELATIONS FOR ABSOLUTE SUMMABILITY
}

\section{B. E. RHOADES and EKREM SAVAŞ}

Received 15 March 2001 and in revised form 15 November 2001

We obtain necessary and (different) sufficient conditions for a series summable $\left|\bar{N}, p_{n}\right|_{k}$, $1<k \leq s<\infty$, to imply that the series is summable $|T|_{s}$, where $\left(\bar{N}, p_{n}\right)$ is a weighted mean matrix and $T$ is a lower triangular matrix. As corollaries of this result, we obtain several inclusion theorems.

2000 Mathematics Subject Classification: 40F05, 40D25, 40G99.

Let $\sum a_{n}$ be a given series with partial sums $s_{n},(C, \alpha)$ the Césaro matrix of order $\alpha$. If $\sigma_{n}^{\alpha}$ denotes the $n$th term of the $(C, \alpha)$-transform of $\left\{s_{n}\right\}$ then, from Flett [4], $\sum a_{n}$ is said to be summable $|C, \alpha|_{k}, k \geq 1$ if

$$
\sum_{n=1}^{\infty} n^{k-1}\left|\sigma_{n}^{\alpha}-\sigma_{n-1}^{\alpha}\right|^{k}<\infty
$$

For any sequence $\left\{u_{n}\right\}$, the forward difference operator $\Delta$ is defined by $\Delta u_{n}=u_{n}-$ $u_{n+1}$.

An appropriate extension of (1) to arbitrary lower triangular matrices $T$ is

$$
\sum_{n=1}^{\infty} n^{k-1}\left|\Delta t_{n-1}\right|^{k}<\infty
$$

where

$$
t_{n}:=\sum_{k=0}^{n} t_{n k} s_{k} .
$$

Such an extension is used, for example, in Bor [2]. But Sarigöl, Sulaiman, and Bor and Thorpe [3] make the following extension of (1).

They define a series $\sum a_{n}$ to be summable $\left|\bar{N}, p_{n}\right|_{k}, k \geq 1$ if

$$
\sum_{n=1}^{\infty}\left(\frac{P_{n}}{p_{n}}\right)^{k-1}\left|\Delta Z_{n-1}\right|^{k}<\infty,
$$

where $Z_{n}$ denotes the $n$th term of the weighted mean transform of $\left\{s_{n}\right\}$; that is,

$$
Z_{n}=\frac{1}{P_{n}} \sum_{k=0}^{n} p_{k} s_{k} .
$$

Apparently they have interpreted the $n$ in (1) to represent the reciprocal of the $n$th diagonal term of the matrix $\left(\bar{N}, p_{n}\right)$. (See, e.g., Sarigöl [6], where this is explicitly the case.) 
Unfortunately, this interpretation cannot be correct. For if it were, then, since the $n$th diagonal entry of $(C, \alpha)$ is $O\left(n^{-\alpha}\right),(1)$ would take the form

$$
\sum_{n=1}^{\infty}\left(n^{\alpha}\right)^{(k-1)}\left|\sigma_{n}^{\alpha}-\sigma_{n-1}^{\alpha}\right|^{k}<\infty .
$$

However, Flett stays with (1). Thus (2) is an appropriate extension of (1) to lower triangular matrices.

Given any lower triangular matrix $T$, we can associate the matrices $\bar{T}$ and $\hat{T}$ with entries defined by

$$
\bar{t}_{n k}=\sum_{i=k}^{n} t_{n i}, \quad \hat{t}_{n k}=\bar{t}_{n k}-\bar{t}_{n-1, k}
$$

respectively.

Thus, from (3),

$$
\begin{aligned}
& t_{n}=\sum_{k=0}^{n} t_{n k} s_{k}=\sum_{k=0}^{n} t_{n k} \sum_{i=0}^{k} a_{i}=\sum_{i=0}^{n} a_{i} \sum_{k=i}^{n} t_{n k}=\sum_{i=0}^{n} \bar{t}_{n k} a_{i}, \\
& Y_{n}:=t_{n}-t_{n-1}=\sum_{i=0}^{n} \bar{t}_{n i} a_{i}-\sum_{i=0}^{n-1} \bar{t}_{n-1, i} a_{i}=\sum_{i=0}^{n} \hat{t}_{n i} a_{i}, \quad \text { since } \bar{t}_{n-1, n}=0 .
\end{aligned}
$$

For a weighted mean matrix $A=\left(\bar{N}, p_{n}\right)$,

$$
\bar{a}_{n k}=\sum_{i=k}^{n} \frac{p_{k}}{P_{n}}=\frac{1}{P_{n}}\left(P_{n}-P_{k-1}\right)=1-\frac{P_{k-1}}{P_{n}} .
$$

Thus

$$
\hat{a}_{n k}=\bar{a}_{n k}-\bar{a}_{n-1, k}=1-\frac{P_{k-1}}{P_{n}}-1+\frac{P_{k-1}}{P_{n-1}}=\frac{p_{n} P_{k-1}}{P_{n} P_{n-1}},
$$

so that, from (5),

$$
X_{n}:=\Delta Z_{n-1}=\frac{p_{n}}{P_{n} P_{n-1}} \sum_{k=0}^{n-1} P_{k-1} a_{k}=\frac{p_{n}}{P_{n} P_{n-1}} \sum_{\nu=1}^{n-1} P_{\nu-1} a_{v},
$$

since $P_{-1}=0$.

We will always assume that $\left\{p_{n}\right\}$ is a positive sequence with $P_{n} \rightarrow \infty$. Also, $\Delta_{v} \hat{t}_{n v}:=$ $\hat{t}_{n v}-\hat{t}_{n, v+1}$.

THEOREM 1. Let $1<k \leq s<\infty,\left\{p_{n}\right\}$ satisfying

$$
\sum_{n=v+1}^{\infty} n^{k-1}\left(\frac{p_{n}}{P_{n} P_{n-1}}\right)^{k}=O\left(\frac{1}{P_{v}^{k}}\right) .
$$

Let $T$ be a lower triangular matrix. Then, the necessary conditions for $\sum a_{n}$ summable $\left|\bar{N}, p_{n}\right|_{k}$ to imply $\sum a_{n}$ is summable $|T|_{s}$ are

(i) $P_{v}\left|t_{v v}\right| / p_{v}=O\left(v^{1 / s-1 / k}\right)$;

(ii) $\sum_{n=v+1}^{\infty} n^{s-1}\left|\Delta_{v} \hat{t}_{n v}\right|^{s}=O\left(v^{s-s / k}\left(p_{v} / P_{v}\right)^{s}\right)$;

(iii) $\sum_{n=v+1}^{\infty} n^{s-1}\left|\hat{t}_{n, v+1}\right|^{s}=O(1)$. 
Proof. We are given that

$$
\sum_{n=1}^{\infty} n^{s-1}\left|Y_{n}\right|^{s}<\infty
$$

whenever

$$
\sum_{n=1}^{\infty} n^{k-1}\left|X_{n}\right|^{k}<\infty .
$$

Now, the space of sequences $\left\{a_{n}\right\}$ satisfying (14) is a Banach space if normed by

$$
\|X\|=\left(\left|X_{0}\right|^{k}+\sum_{n=1}^{\infty} n^{k-1}\left|X_{n}\right|^{k}\right)^{1 / k} .
$$

We also consider the space of those sequences $\left\{Y_{n}\right\}$ that satisfy (13). This is also a BK-space with respect to the norm

$$
\|Y\|=\left(\left|Y_{0}\right|^{k}+\sum_{n=1}^{\infty} n^{s-1}\left|Y_{n}\right|^{s}\right)^{1 / s} .
$$

Observe that (8) transforms the space of sequences satisfying (14) into the space of sequences satisfying (13). Applying the Banach-Steinhaus theorem, there exists a constant $K>0$ such that

$$
\|Y\| \leq K\|X\| .
$$

Applying (11) and (8) to $a_{v}=e_{v}-e_{v+1}$, where $e_{v}$ is the $v$ th coordinate vector, we have, respectively,

$$
\begin{gathered}
X_{n}= \begin{cases}0, & \text { if } n<v, \\
\frac{p_{v}}{P_{v}}, & \text { if } n=v, \\
-\frac{p_{v} p_{n}}{P_{n} P_{n-1}}, & \text { if } n>v,\end{cases} \\
Y_{n}= \begin{cases}0, & \text { if } n<v, \\
\hat{t}_{n v}, & \text { if } n=v, \\
\Delta_{v} \hat{t}_{n v}, & \text { if } n>v .\end{cases}
\end{gathered}
$$

By (15) and (16), it follows that

$$
\begin{gathered}
\|X\|=\left\{v^{k-1}\left(\frac{p_{v}}{P_{v}}\right)^{k}+\sum_{n=v+1}^{\infty} n^{k-1}\left(\frac{p_{v} p_{n}}{P_{n} P_{n-1}}\right)^{k}\right\}^{1 / k}, \\
\|Y\|=\left\{v^{s-1}\left|t_{v v}\right|^{s}+\sum_{n=v+1}^{\infty} n^{s-1}\left|\Delta_{v} \hat{t}_{n v}\right|^{s}\right\}^{1 / s},
\end{gathered}
$$

recalling that $\hat{t}_{v v}=\bar{t}_{v v}=t_{v v}$. 
Using (19) and (20) in (17), along with (12), it follows that

$$
\begin{aligned}
v^{s-1}\left|t_{v v}\right|^{s}+\sum_{n=v+1}^{\infty} n^{s-1}\left|\Delta \hat{t}_{n v}\right|^{s} & \leq K^{s}\left(v^{k-1}\left(\frac{p_{v}}{P_{v}}\right)^{k}+\sum_{n=v+1}^{\infty} n^{k-1}\left(\frac{p_{v} p_{n}}{P_{n} P_{n-1}}\right)^{k}\right)^{s / k} \\
& \leq K^{s}\left(v^{k-1}\left(\frac{p_{v}}{P_{v}}\right)^{k}+O(1)\left(\frac{p_{v}}{P_{v}}\right)^{k}\right)^{s / k} \\
& =O\left(\left(\frac{p_{v}}{P_{v}}\right)^{k} v^{k-1}\right)^{s / k} .
\end{aligned}
$$

The above inequality will be true if and only if each term on the left-hand side is $O\left(\left(p_{v} / P_{v}\right)^{k} v^{k-1}\right)^{s / k}$.

Taking the first term,

$$
\begin{aligned}
v^{s-1}\left|t_{v v}\right|^{s} & =O\left(\left(\frac{p_{v}}{P_{v}}\right)^{k} v^{k-1}\right)^{s / k}, \\
\left|t_{v v}\right|^{s} & =O\left(\left(\frac{p_{v}}{P_{v}}\right)^{s} v^{1-s / k}\right), \\
\left|t_{v v}\right| & =O\left(\left(\frac{p_{v}}{P_{v}}\right)^{s} v^{1-s / k}\right)^{1 / s} \\
& =O\left(\left(\frac{p_{v}}{P_{v}}\right) v^{1 / s-1 / k}\right),
\end{aligned}
$$

which verifies that (i) is necessary.

Using the second term, we have

$$
\sum_{n=v+1}^{\infty} n^{s-1}\left|\Delta \hat{t}_{n v}\right|^{s}=O\left(\left(\frac{p_{v}}{P_{v}}\right)^{k} v^{k-1}\right)^{s / k}=O\left(\left(\frac{p_{v}}{P_{v}}\right)^{s} v^{s-s / k}\right)
$$

which is condition (ii).

If we now apply (11) and (8) to $a_{v}=e^{v+1}$, we have, respectively,

$$
\begin{gathered}
X_{n}= \begin{cases}0, & \text { if } n \leq v, \\
\frac{P_{v} p_{n}}{P_{n} P_{n-1}}, & \text { if } n>v,\end{cases} \\
Y_{n}= \begin{cases}0, & \text { if } n \leq v, \\
\hat{t}_{n, v+1}, & \text { if } n>v .\end{cases}
\end{gathered}
$$


The corresponding norms are

$$
\begin{aligned}
& \|X\|=\left\{\sum_{n=v+1}^{\infty} n^{k-1}\left(\frac{P_{v} p_{n}}{P_{n} P_{n-1}}\right)^{k}\right\}^{1 / k}, \\
& \|Y\|=\left\{\sum_{n=v+1}^{\infty} n^{s-1}\left|\hat{t}_{n, v+1}\right|^{s}\right\}^{1 / s} .
\end{aligned}
$$

Applying (17) and (12),

$$
\sum_{n=v+1}^{\infty} n^{s-1}\left|\hat{t}_{n, v+1}\right|^{s} \leq K^{s}\left\{\sum_{n=v+1}^{\infty} n^{k-1}\left(\frac{P_{v} p_{n}}{P_{n} P_{n-1}}\right)^{k}\right\}^{s / k},
$$

which is condition (iii).

COROLlaRY 2. Let $T$ be a lower triangular matrix, $\left\{p_{n}\right\}$ satisfying (12). Then the necessary conditions for $\sum a_{n}$ summable $\left|\bar{N}, p_{n}\right|_{k}$ to imply $\sum a_{n}$ summable $|T|_{k}$ are

(i) $P_{v}\left|t_{v v}\right| / p_{v}=O(1)$;

(ii) $\sum_{n=v+1}^{\infty} n^{k-1}\left|\Delta_{v} \hat{t}_{n v}\right|^{k}=O\left(v^{k-1}\left(p_{v} / P_{v}\right)^{k}\right)$;

(iii) $\sum_{n=v+1}^{\infty} n^{k-1}\left|\hat{t}_{n, v+1}\right|^{k}=O(1)$.

To prove Corollary 2, simply set $s=k$ in Theorem 1 .

A lower triangular matrix $T$ is called a triangle if each $t_{n n} \neq 0$.

THEOREM 3. Let $1<k \leq s<\infty$. Let $T$ be a triangle with bounded entries such that $T$ and $\left\{p_{n}\right\}$ satisfy the following:

(i) $t_{v v}=O\left(\left(p_{v} / P_{v}\right) v^{1 / s-1 / k}\right)$;

(ii) $\left(n\left|X_{n}\right|\right)^{s-k}=O(1)$;

(iii) $\sum_{v=1}^{n-1}\left|\Delta_{v} \hat{t}_{n v}\right|=O\left(\left|t_{n n}\right|\right)$;

(iv) $\sum_{n=v+1}^{\infty}\left(n\left|t_{n n}\right|\right)^{s-1}\left|\Delta_{v} \hat{t}_{n v}\right|=O\left(\nu^{s-1}\left|t_{v v}\right|^{s}\right)$;

(v) $\sum_{v=1}^{n-1}\left|t_{v v}\right|\left|\hat{t}_{n, v+1}\right|=O\left(\left|t_{n n}\right|\right)$;

(vi) $\sum_{n=v+1}^{\infty}\left(n\left|t_{n n}\right|\right)^{s-1}\left|\hat{t}_{n, v+1}\right|=O\left(v\left|t_{v v}\right|\right)^{s-1}$.

Then $\sum a_{n}$ is $\bar{N},\left.p_{n}\right|_{k}$.

Proof. Solving (11) for $\left\{a_{n}\right\}$ and substituting into (8) give

$$
\begin{aligned}
Y_{n} & =\sum_{v=1}^{n} \hat{t}_{n v}\left(\frac{X_{v} P_{v}}{p_{v}}-\frac{X_{v-1} P_{v-2}}{p_{v-1}}\right) \\
& =\sum_{v=1}^{n} \hat{t}_{n v} \frac{X_{v} P_{v}}{p_{v}}-\sum_{v=1}^{n} \hat{t}_{n v} \frac{X_{v-1} P_{v-2}}{p_{v-1}} \\
& =\sum_{v=1}^{n} \hat{t}_{n v} \frac{X_{v} P_{v}}{p_{v}}-\sum_{v=0}^{n-1} \hat{t}_{n, v+1} \frac{X_{v} P_{v-1}}{p_{v}} \\
& =\frac{\hat{t}_{n n} X_{n} P_{n}}{p_{n}}+\sum_{v=1}^{n-1}\left(\hat{t}_{n v} P_{v}-\hat{t}_{n, v+1} P_{v-1}\right) \frac{X_{v}}{p_{v}}
\end{aligned}
$$




$$
\begin{aligned}
& =\frac{t_{n n} P_{n} X_{n}}{p_{n}}+\sum_{v=1}^{n-1}\left[P_{v}\left(\hat{t}_{n v}-\hat{t}_{n, v+1}\right)+\hat{t}_{n, v+1}\left(P_{v}-P_{v-1}\right)\right] \frac{X_{v}}{p_{v}} \\
& =\frac{P_{n} t_{n n} X_{n}}{p_{n}}+\sum_{v=1}^{n-1}\left(\frac{P_{v}}{p_{v}} \Delta_{v} \hat{t}_{n v}+\hat{t}_{n, v+1}\right) X_{v} \\
& =T_{n 1}+T_{n 2}+T_{n 3} .
\end{aligned}
$$

From Minkowski's inequality, it is sufficient to show that

$$
\sum_{n=1}^{\infty} n^{s-1}\left|T_{n i}\right|^{s}<\infty, \quad i=1,2,3 .
$$

Using condition (i) of Theorem 3,

$$
\begin{aligned}
J_{1} & :=\sum_{n=1}^{\infty} n^{s-1}\left|T_{n 1}\right|^{s}=\sum_{n=1}^{\infty} n^{s-1}\left|\frac{t_{n n} P_{n}}{p_{n}} X_{n}\right|^{s} \\
& =O(1) \sum_{n=1}^{\infty} n^{s-1}\left(n^{1 / s-1 / k}\right)^{s}\left|X_{n}\right|^{s} \\
& =O(1) \sum_{n=1}^{\infty} n^{k-1}\left|X_{n}\right|^{k}\left(n^{s-s / k-k+1}\left|X_{n}\right|^{s-k}\right) .
\end{aligned}
$$

But

$$
n^{s-s / k-k+1}\left|X_{n}\right|^{s-k}=\left(n^{1-1 / k}\left|X_{n}\right|\right)^{s-k}=O\left(\left(n\left|X_{n}\right|\right)^{s-k}\right)=O(1),
$$

from (ii) of Theorem 3.

Since $\sum a_{n}$ is summable, $\left|\bar{N}, p_{n}\right|_{k}, J_{1}=O(1)$.

Using Hölder's inequality and conditions (i), (ii), (iii), and (iv) of Theorem 3.

$$
\begin{aligned}
J_{2} & :=\sum_{n=1}^{\infty} n^{s-1}\left|T_{n 2}\right|^{s}=\sum_{n=1}^{\infty} n^{s-1}\left|\sum_{v=1}^{n-1}\left(\frac{P_{v}}{p_{v}}\right)\left(\Delta_{v} \hat{t}_{n v}\right) X_{v}\right|^{s} \\
& =O(1) \sum_{n=1}^{\infty} n^{s-1}\left(\sum_{v=1}^{n-1} v^{1 / s-1 / k}\left|t_{v v}\right|^{-1}\left|\Delta_{v} \hat{t}_{n v}\right|\left|X_{v}\right|\right)^{s} \\
& =O(1) \sum_{n=1}^{\infty} n^{s-1}\left(\sum_{v=1}^{n-1} v^{1-s / k}\left|t_{v v}\right|^{-s}\left|\Delta_{v} \hat{t}_{n v}\right|\left|X_{v}\right|^{s}\right) \times\left(\sum_{v=1}^{n-1}\left|\Delta_{v} \hat{t}_{n v}\right|\right)^{s-1} \\
& =O(1) \sum_{n=1}^{\infty}\left(n\left|t_{n n}\right|\right)^{s-1} \sum_{v=1}^{n-1} v^{1-s / k}\left|t_{v v}\right|^{-s}\left|\Delta_{v} \hat{t}_{n v}\right|\left|X_{v}\right|^{s} \\
& =O(1) \sum_{v=1}^{\infty} v^{1-s / k}\left|t_{v v}\right|^{-s}\left|X_{v}\right|^{s} \sum_{n=v+1}^{\infty}\left(n\left|t_{n n}\right|\right)^{s-1}\left|\Delta_{v} \hat{t}_{n v}\right|
\end{aligned}
$$




$$
\begin{aligned}
& =O(1) \sum_{\nu=1}^{\infty} v^{1-s / k}\left|t_{v v}\right|^{-s}\left|X_{v}\right|^{s} v^{s-1}\left|t_{v v}\right|^{s} \\
& =O(1) \sum_{v=1}^{\infty} v^{s-s / k}\left|X_{v}\right|^{s} \\
& =O(1) \sum_{\nu=1}^{\infty} v^{k-1}\left|X_{v}\right|^{k}\left(v^{s-s / k-k+1}\left|X_{v}\right|^{s-k}\right) \\
& =O(1) \sum_{\nu=1}^{\infty} v^{k-1}\left|X_{v}\right|^{k}=O(1) .
\end{aligned}
$$

By Hölder's inequality and conditions (v), (vi), and (iii) of Theorem 3, we have

$$
\begin{aligned}
J_{3} & :=\sum_{n=1}^{\infty} n^{s-1}\left|T_{n 3}\right|^{s}=\sum_{n=1}^{\infty} n^{s-1}\left|\sum_{v=1}^{n-1} \hat{t}_{n, v+1} X_{v}\right|^{s} \\
& \leq \sum_{n=1}^{\infty} n^{s-1}\left(\sum_{v=1}^{n-1}\left|\hat{t}_{n, v+1}\right|\left|X_{v}\right|\right)^{s} \\
& \leq \sum_{n=1}^{\infty} n^{s-1}\left(\sum_{v=1}^{n-1}\left|t_{v v}\right|^{1-s}\left|\hat{t}_{n, v+1}\right|\left|X_{v}\right|^{s}\right) \\
& =O\left(\sum_{v=1}^{n-1}\left|t_{v v}\right|\left|\hat{t}_{n, v+1}\right|\right)^{s-1} \sum_{n=1}^{\infty}\left(n\left|t_{n n}\right|\right)^{s-1} \sum_{v=1}^{n-1}\left|t_{v v}\right|^{1-s}\left|\hat{t}_{n, v+1}\right|\left|X_{v}\right|^{s} \\
& =O(1) \sum_{v=1}^{\infty}\left|t_{v v}\right|^{1-s}\left|X_{v}\right|^{s} \sum_{n=v+1}^{\infty}\left(n\left|t_{n n}\right|\right)^{s-1}\left|\hat{t}_{n, v+1}\right| \\
& =O(1) \sum_{v=1}^{\infty}\left|t_{v v}\right|^{1-s}\left|X_{v}\right|^{s}\left(v\left|t_{v v}\right|\right)^{s-1} \\
& =O(1) \sum_{v=1}^{\infty} v^{s-1}\left|X_{v}\right|^{s} \\
& =O(1) \sum_{v=1}^{\infty} v^{k-1}\left|X_{v}\right|^{k}\left(v\left|X_{v}\right|\right)^{s-k} \\
& =O(1) \sum_{v=1}^{\infty} v^{k-1}\left|X_{v}\right|^{k}=O(1) .
\end{aligned}
$$

COROLlary 4 (see [5]). Let $T$ be a nonnegative lower triangular matrix, $\left\{p_{n}\right\} a$ positive sequence satisfying

(i) $t_{n i} \geq t_{n+1, i}, n \geq i, i=0,1,2, \ldots$;

(ii) $P_{n} t_{n n}=O\left(p_{n}\right)$;

(iii) $\bar{t}_{n 0}=\bar{t}_{n-1,0}, n=1,2, \ldots$; 
(iv) $\sum_{i=1}^{n-1}\left|t_{i i}\right|\left|\hat{t}_{n, i+1}\right|=O\left(t_{n n}\right)$;

(v) $\sum_{n=i+1}^{\infty}\left(n t_{n n}\right)^{k-1}\left|\Delta_{i} \hat{t}_{n i}\right|=O\left(i^{k-1} t_{i i}^{k}\right)$;

(vi) $\sum_{n=i+1}^{\infty}\left(n t_{n n}\right)^{k-1}\left|\hat{t}_{n, i+1}\right|=O\left(\left(i t_{i i}\right)^{k-1}\right)$.

Then $\sum a_{n}$ summable $\left|\bar{N}, p_{n}\right|_{k}$ implies $\sum a_{n}$ summable $|T|_{k}, k \geq 1$.

Proof. Since $s=k$ and $T$ is nonnegative, condition (ii) of Theorem 3 is automatically satisfied, and conditions (ii), (iv), (v), and (vi) of Corollary 4 are equivalent to conditions (i), (v), (iv), and (vi) of Theorem 3, respectively

$$
\Delta_{v} \hat{t}_{n v}=\hat{t}_{n v}-\hat{t}_{n, v+1}=\bar{t}_{n v}-\bar{t}_{n-1, v}-\bar{t}_{n, v+1}+\bar{t}_{n-1, v+1}=t_{n v}-t_{n-1, v} .
$$

Therefore, using conditions (i) and (iii) of Corollary 4,

$$
\sum_{v=1}^{n-1}\left|\Delta_{v} \hat{t}_{n v}\right|=\sum_{v=1}^{n-1}\left(t_{n-1, v}-t_{n v}\right)=1-t_{n-1,0}-1+t_{n n}+t_{n 0} \leq t_{n n},
$$

and condition (iii) of Theorem 3 is satisfied.

REMARK 5. For $1<k \leq s<\infty$, necessary and sufficient conditions for a triangle $A: \ell^{k} \rightarrow \ell^{s}$ are known only for factorable matrices (see Bennett [1]), which include weighted mean matrices. Therefore, we should not expect to obtain a set of necessary and sufficient conditions when an arbitrary triangle is involved.

However, necessary and sufficient conditions for a matrix $A: \ell \rightarrow \ell^{s}, 1 \leq s<\infty$ are known. The following result comes from Theorem 2.1 of Rhoades and Savaş [5] by setting each $\lambda_{n}=1$.

THEOREM 6. Let $T$ be a lower triangular matrix. Then $\sum a_{n}$ summable $\left|\bar{N}, p_{n}\right|_{k}$ implies $\sum a_{n}$ summable $|T|_{s}, s \geq 1$ if and only if

(i) $P_{v}\left|t_{v v}\right| / p_{v}=O\left(v^{1 / s-1}\right)$,

(ii) $\sum_{n=v+1}^{\infty} n^{s-1}\left|\Delta_{v} \hat{t}_{n v}\right|^{s}=O\left(\left(p_{v} / P_{v}\right)^{s}\right)$,

(iii) $\sum_{n=v+1}^{\infty} n^{s-1}\left|\hat{t}_{n, v+1}\right|^{s}=O(1)$.

REMARK 7. In [5], it is assumed that $T$ has nonnegative entries and row sums one, but these restrictions are not used in the proofs.

Finally, we state necessary and sufficient conditions when $k=s=1$.

THEOREM 8. The series $\sum a_{n}$ summable $\left|\bar{N}, p_{n}\right|$ implies $\sum a_{n}$ summable $T$ if and only if

(i) $P_{v}\left|t_{v v}\right| / p_{v}=O(1)$;

(ii) $\sum_{n=v+1}^{\infty}\left|\Delta_{v} \hat{t}_{n v}\right|=O\left(p_{v} / P_{v}\right)$;

(iii) $\sum_{n=v+1}^{\infty}\left|\hat{t}_{n, v+1}\right|=O(1)$.

Proof. Note that, with $k=1,(12)$ is automatically satisfied. Therefore, the necessity of the conditions follows from Theorem 1 .

To prove the conditions sufficient, use [5, Corollary 4.1] by setting each $\lambda_{n}=1$.

COROLLARY 9. $\sum a_{n}$ summable $|C, 1|$ implies $\sum a_{n}\left|\bar{N}, q_{n}\right|$ if and only if

(i) $n q_{n} / Q_{n}=O(1)$. 
Proof. With each $p_{n}=1, T=\left(\bar{N}, q_{n}\right)$, condition (i) of Theorem 8 reduces to condition (i) of Corollary 9.

Using (33),

$$
\begin{aligned}
\sum_{n=v+1}^{\infty}\left|\Delta_{v} \hat{t}_{n v}\right| & =\sum_{n=v+1}^{\infty}\left|t_{n v}-t_{n-1, v}\right|=\sum_{n=v+1}^{\infty}\left|\frac{p_{v}}{P_{n}}-\frac{p_{v}}{P_{n-1}}\right| \\
& =p_{v} \sum_{n=v+1}^{\infty} \frac{p_{n}}{P_{n} P_{n-1}}=\frac{p_{v}}{P_{v}}
\end{aligned}
$$

and condition (ii) of Theorem 8 is satisfied. Since $\left(\bar{N}, p_{n}\right)$ has row sums one,

$$
\begin{aligned}
\hat{t}_{n, v+1} & =\bar{t}_{n, v+1}-\bar{t}_{n-1, v+1}=\sum_{i=v+1}^{n} t_{n i}-\sum_{i=v+1}^{n} t_{n-1, i} \\
& =1-\sum_{i=0}^{v} t_{n i}-1+\sum_{i=0}^{v} t_{n-1, i} \\
& =\sum_{i=0}^{v}\left(t_{n-1, i}-t_{n i}\right)=\sum_{i=0}^{v}\left(\frac{p_{i}}{P_{n-1}}-\frac{p_{i}}{P_{n}}\right) \\
& =\frac{p_{n}}{P_{n} P_{n-1}} \sum_{i=0}^{v} p_{i}=\frac{p_{n} P_{v}}{P_{n} P_{n-1}} .
\end{aligned}
$$

Therefore

$$
\sum_{n=v+1}^{\infty}\left|\hat{t}_{n, v+1}\right|=P_{v} \sum_{n=v+1}^{\infty} \frac{p_{n}}{P_{n} P_{n-1}}=1,
$$

and condition (iii) of Theorem 8 is satisfied.

COROLlary 10. The series $\sum a_{n}$ summable $\left|\bar{N}, p_{n}\right|_{k}$ implies $\sum a_{n}$ summable $|C, 1|_{k}$ if and only if

(i) $P_{n} /\left(n p_{n}\right)=O(1)$.

Proof. Using $T=(C, 1)$ in Theorem 8 , condition (i) of Theorem 8 reduces to condition (i) of Corollary 10.

From (33) and (i) of Corollary 10,

$$
\begin{aligned}
\sum_{n=v+1}^{\infty}\left|\Delta_{v} \hat{t}_{n v}\right| & =\sum_{n=v+1}^{\infty}\left|t_{n-1, v}-t_{n v}\right|=\sum_{n=v+1}^{\infty}\left(\frac{1}{n}-\frac{1}{n+1}\right) \\
& =\frac{1}{v+1}=\frac{P_{v}}{v p_{v}}\left(\frac{v}{v+1}\right)\left(\frac{p_{v}}{P_{v}}\right)=O\left(\frac{p_{v}}{P_{v}}\right),
\end{aligned}
$$

and condition (ii) of Theorem 8 is satisfied. 
Using (36),

$$
\begin{aligned}
\sum_{n=v+1}^{\infty}\left|\hat{t}_{n, v+1}\right| & =\sum_{n=v+1}^{\infty}\left|\sum_{i=0}^{v}\left(t_{n-1, i}-t_{n i}\right)\right| \\
& =\sum_{n=v+1}^{\infty}\left|\sum_{i=0}^{v}\left(\frac{1}{n}-\frac{1}{n+1}\right)\right| \\
& =\sum_{n=v+1}^{\infty}\left(\frac{1}{n}-\frac{1}{n+1}\right)(v+1)=(v+1)\left(\frac{1}{v+1}\right)=1,
\end{aligned}
$$

and condition (iii) of Theorem 8 is satisfied.

Combining Corollaries 9 and 10, we have the following corollary.

COROLLARY 11. $\left|\bar{N}, p_{n}\right|$ and $|C, 1|$ are equivalent if and only if

(i) $n p_{n} / P_{n}=O(1)$;

(ii) $P_{n} /\left(n p_{n}\right)=O(1)$.

ACKNOWLEDGMENT. The first author received partial support from the Scientific and Technical Research Council of Turkey during the preparation of this paper.

\section{REFERENCES}

[1] G. Bennett, Some elementary inequalities, Quart. J. Math. Oxford Ser. (2) 38 (1987), no. 152, 401-425.

[2] H. Bor, On the relative strength of two absolute summability methods, Proc. Amer. Math. Soc. 113 (1991), no. 4, 1009-1012.

[3] H. Bor and B. Thorpe, A note on two absolute summability methods, Analysis 12 (1992), no. 1-2, 1-3.

[4] T. M. Flett, On an extension of absolute summability and some theorems of Littlewood and Paley, Proc. London Math. Soc. (3) 7 (1957), 113-141.

[5] B. E. Rhoades and E. Savaş, A characterization of absolute summability factors, submitted to Taiwanese J. Math.

[6] M. A. Sarigöl, On local property of $|A|_{k}$ summability of factored Fourier series, J. Math. Anal. Appl. 188 (1994), no. 1, 118-127.

B. E. RHoAdes: Department of MATHEMATics, Indiana UniVersity, BloOMington, IN 47405-7106, USA

E-mail address: rhoades@indiana.edu

EKREM SAVAŞ: DePARTMENT OF MATHEMATICS, YÜZÜNCÜ YIL UNIVERSITY, VAN, TURKeY

E-mail address: ekremsavas@yahoo.com 


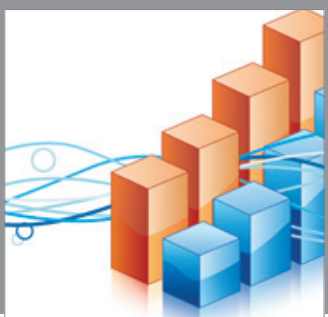

Advances in

Operations Research

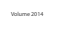

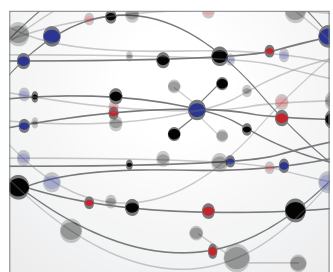

\section{The Scientific} World Journal
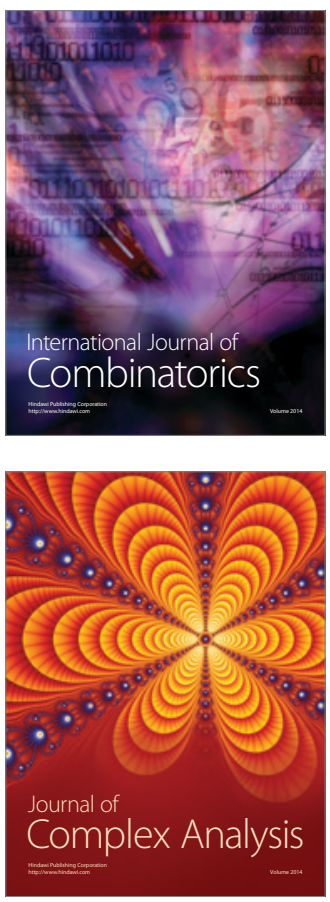

International Journal of

Mathematics and

Mathematical

Sciences
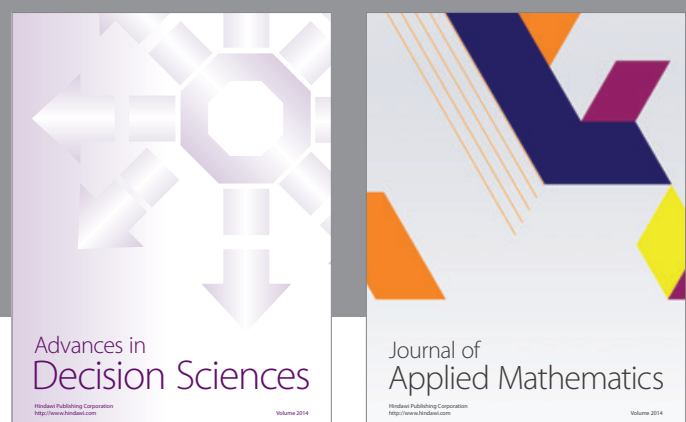

Journal of

Applied Mathematics
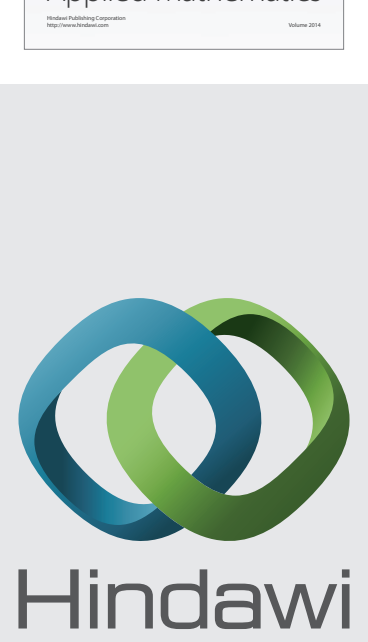

Submit your manuscripts at http://www.hindawi.com
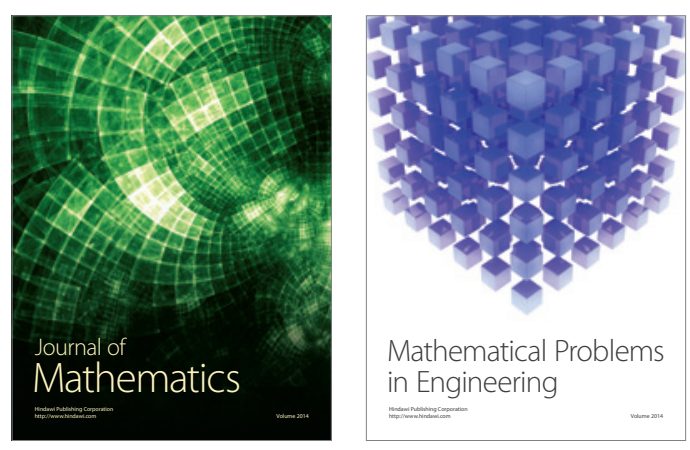

Mathematical Problems in Engineering
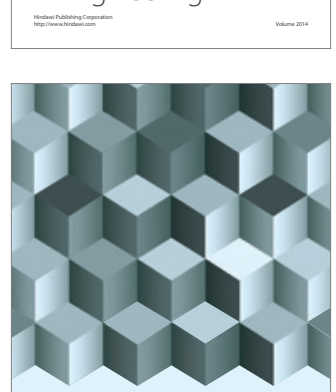

Journal of

Function Spaces
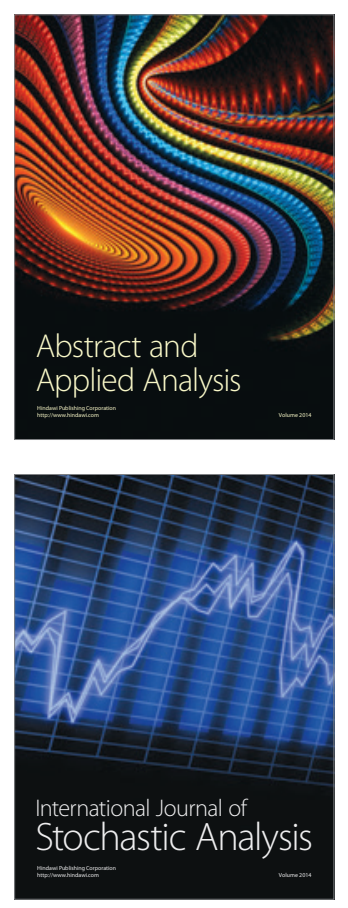

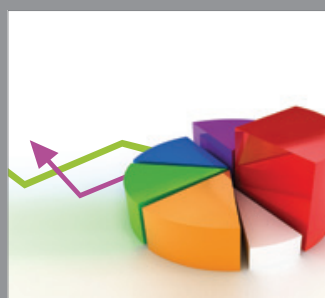

ournal of

Probability and Statistics

Promensencen
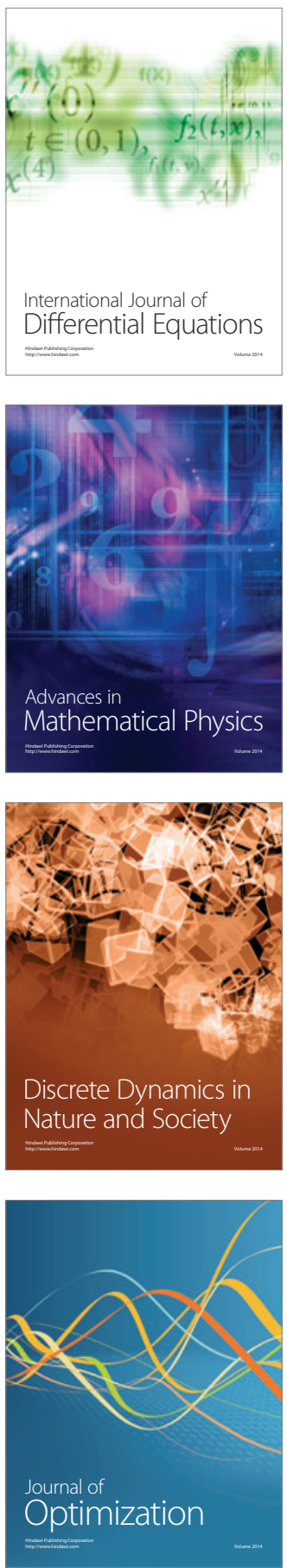